PROCEEDINGS OF THE AMERICAN MATHEMATICAL SOCIETY

Volume 124, Number 7, July 1996

\title{
ON THE DIMENSION OF INFINITE COVERS
}

\author{
W. G. DWYER, S. STOLZ, AND L. R. TAYLOR
}

(Communicated by Thomas Goodwillie)

\begin{abstract}
We prove the following theorem and some generalizations.
\end{abstract}
Theorem A. Let $X$ be a connected $C W$ complex which satisfies Poincaré duality of dimension $n \geq 4$. For any subgroup $H$ of $\pi_{1}(X)$, let $X_{H}$ denote the cover of $X$ corresponding to $H$. If $H$ has infinite index in $\pi_{1}(X)$, then $X_{H}$ is homotopy equivalent to an $(n-1)$-dimensional $C W$ complex.

If $X$ is also a $K(\pi, 1)$, Theorem A is a result of Strebel [St]. The lack of such a theorem in general is lamented in $[\mathrm{H}]$, and Theorem A allows the extension of Theorem 2 (p. 157) and its Corollary (p. 158) in [H] from 4-dimensional manifolds to 4-dimensional Poincaré spaces. We begin with some notation and propositions.

Let $R$ be a commutative ring, $G$ a discrete group, and $R G$ the group ring. We say that a chain complex of left $R G$ modules, $E_{*}$, satisfies condition $H D(n)$ iff $H^{r}\left(E_{*} ; M\right)=0$ for all left $R G$ modules, $M$, and all $r>n$. Wall's techniques [W] can be used to show that $E_{*}$ satisfies condition $H D(n)$ iff $E_{*}$ is chain homotopy equivalent to a complex $E_{*}^{\prime}$ with $E_{r}^{\prime}=0, r>n$, by maps $f_{r}: E_{r}^{\prime} \rightarrow E_{r}$ and $g_{r}: E_{r} \rightarrow E_{r}^{\prime}$ such that $g_{r} f_{r}=1_{E_{r}^{\prime}}$ for all $r$, and $f_{r} g_{r}=1_{E_{r}}$ for all $r<n$. If $E_{*}$ is a complex of projective modules, Wall's condition $D(n)$ is equivalent to condition $H D(n)$. We say that a connected space satisfies $H D(n)$ iff the singular chain complex of its universal cover, $S_{*}(\tilde{X})$, considered as a complex of $\mathbb{Z} \pi_{1}(X)$ modules, does. In particular, if $n \neq 2$, it follows from Wall [W] that a CW complex, $X$, satisfies $H D(n)$ iff $X$ is homotopy equivalent to an $n$-dimensional complex.

Given a subgroup $H \subset G$ and a chain complex $E_{*}$ on left $R G$-modules, we can consider $E_{*}$ as a chain complex of $R H$ modules by restriction: we write $R H \mid E_{*}$ for this complex. Let $\mathcal{C}_{H}{ }^{R}{ }_{G}$ denote the collection of all right $R G$ modules $D$ such that $D \otimes_{R G} \operatorname{Hom}_{R H}(R G, L)=0$ for all left $R H$ modules $L$. Given any left $R G$ module $M$ and integer $r$, there exists a natural map $\mu_{M}[r]: H^{r}\left(E_{*} ; R G\right) \otimes_{R G}$ $M \rightarrow H^{r}\left(E_{*} ; M\right)$ induced by the maps $\operatorname{Hom}_{R G}\left(E_{r}, R G\right) \otimes_{R G} M \rightarrow \operatorname{Hom}_{R G}\left(E_{r}, M\right)$ defined by $\gamma \otimes m \mapsto(e \mapsto \gamma(e) \cdot m)$.

Throughout this paper our conventions are the same as those in [CE], Chapter 2. The notation $\operatorname{Hom}_{S}(A, B)$ will only be used if $A$ and $B$ are left $S$ modules and $C \otimes_{S} B$ will be used if $C$ is a right $S$ module and $B$ is a left $S$ module. Both $\operatorname{Hom}_{S}$ and $\otimes_{S}$ have additional module structure when $A, B$, or $C$ has a bimodule structure. If $H$ and $K$ are subgroups of $G$, then $R G$ is a $R H-R K$ bimodule and

Received by the editors May 10, 1994 and, in revised form, November 18, 1994.

1991 Mathematics Subject Classification. Primary 55U15, 57P10.

Key words and phrases. Infinite covers, dimension.

Partially supported by the National Science Foundation. 
this is the source of all module structure on tensor products, homology groups, cohomology groups, etc., occurring here.

Proposition 1. Let $E_{*}$ satisfy condition $H D(n)$. If $\mu_{M}[n]$ is onto for all left $R G$ modules $M$ and if $H^{n}\left(E_{*} ; R G\right) \in \mathcal{C}_{H}{ }^{R}{ }_{G}$, then ${ }_{R H} \mid E_{*}$ satisfies $H D(n-1)$. If $\mu_{M}[n]$ is injective for all left $R G$ modules $M$ and if $R H \mid E_{*}$ satisfies $H D(n-1)$, then $H^{n}\left(E_{*} ; R G\right) \in \mathcal{C}_{H}{ }_{G}$.

The next few results are useful for finding $R G$ modules that are in $\mathcal{C}_{H}{ }^{R}$.

Proposition 2. The following are equivalent.

(2.1) The map $\lambda_{H}: D \rightarrow D \otimes_{R G} \operatorname{Hom}_{R H}(R G, R G)$ with $\lambda_{H}(d)=d \otimes 1_{R G}$ is the zero map.

(2.2) $D \otimes_{R G} \operatorname{Hom}_{R H}(R G, R G)=0$.

(2.3) $D \in \mathcal{C}_{H}{ }_{G}$.

Here are some ways to construct elements in $\mathcal{C}_{H}{ }^{R}{ }_{G}$ from other elements.

Proposition 3. The following results hold.

(3.1) If $H \subset G^{\prime} \subset G$ and if $\left|G: G^{\prime}\right|<\infty$, then for any $R G$ module $D,\left.D\right|_{R G^{\prime}} \in$ $\mathcal{C}_{H}{ }^{R}{ }_{G^{\prime}}$ iff $D \in \mathcal{C}_{H}{ }^{R}{ }_{G}$.

(3.2) If $H^{\prime} \subset H \subset G, \mathcal{C}_{H}{ }^{R}{ }_{G} \subset \mathcal{C}_{H^{\prime}}{ }^{R}{ }_{G}$ : if $\left|H: H^{\prime}\right|<\infty$, then $\mathcal{C}_{H^{\prime}}{ }^{R}{ }_{G} \subset \mathcal{C}_{H}{ }^{R}{ }_{G}$.

(3.3) If $D_{1} \rightarrow D_{2} \rightarrow 0$ is exact and if $D_{1} \in \mathcal{C}_{H}{ }_{G}$, then $D_{2} \in \mathcal{C}_{H}{ }_{G}$.

(3.4) If $D_{0} \rightarrow D_{1} \rightarrow D_{2} \rightarrow 0$ is exact, and if $D_{0}, D_{2} \in \mathcal{C}_{H}{ }^{R}{ }_{G}$, then $D_{1} \in \mathcal{C}_{H}{ }^{R}$.

(3.5) For any index set $\mathcal{S}, D_{\alpha} \in \mathcal{C}_{H}{ }^{R}{ }_{G}$ for each $\alpha \in \mathcal{S}$ iff $\left(\bigoplus_{\alpha \in \mathcal{S}} D_{\alpha}\right) \in \mathcal{C}_{H}{ }^{R}{ }_{G}$.

(3.6) If $p: F \rightarrow G$ is an epimorphism; if $D$ is an $R G$ module; and if $D$, considered as an $R F$ module is in $\mathcal{C}_{p^{-1}(H)}{ }^{R}{ }_{F}$, then $D \in \mathcal{C}_{H}{ }^{R}{ }_{G}$.

Let $R^{\text {tr }}$ denote $R$ considered as a trivial $R G$ module. Here are two exercises, 4(b) and 4(c) on page 71, from Ken Brown's book [Brn] showing that $R^{\operatorname{tr}} \in \mathcal{C}_{H}{ }^{R}$.

Proposition 4. $R^{\text {tr }} \in \mathcal{C}_{H}{ }^{R}$ G provided

(4.1) $G$ is finitely generated and $H$ has infinite index in $G$; or more generally

(4.2) there exists a finitely generated subgroup $G^{\prime} \subset G$ such that the index of $G^{\prime} \cap$ $g \mathrm{Hg}^{-1}$ in $\mathrm{G}^{\prime}$ is infinite for all $\mathrm{g} \in \mathrm{G}$.

For any $R$ module $D$, let $\operatorname{Aut}(D)$ denote the $R$ automorphism group of $D$. Let $R^{\times}$denote the group of units of $R$. Multiplication by $r \in R^{\times}$gives an automorphism of $D$ and hence a homomorphism $i: R^{\times} \rightarrow \operatorname{Aut}^{\mathrm{op}}(D)$ : let $P \operatorname{Aut}^{\mathrm{op}}(D)=$ Aut $^{\mathrm{op}}(D) / i\left(R^{\times}\right)$. To give $D$ a right $R G$ module structure is equivalent to giving a homomorphism $s: G \rightarrow \operatorname{Aut}^{\mathrm{op}}(D)$ : let $\hat{s}: G \rightarrow P \operatorname{Aut}^{\mathrm{op}}(D)$ denote the evident composition and let $G_{D}$ denote the kernel of $\hat{s}$.

Proposition 5. The following results hold.

(5.1) If $D=R$ as an $R$ module, then $G_{D}=G$.

If $G_{D}$ has finite index in $G$, then $D \in \mathcal{C}_{H}{ }^{R}$ G provided either

(5.2) $R^{\operatorname{tr}} \in \mathcal{C}_{H}{ }^{R}{ }_{G}$ and $i: R^{\times} \rightarrow \operatorname{Aut}(D)$ is injective; or

(5.3) $p: F \rightarrow G$ is an epimorphism from a free group and $R^{\operatorname{tr}} \in \mathcal{C}_{p^{-1}(H)}{ }_{F}$.

Proof of Theorem A. We prove that $X_{H}$ satisfies $H D(n-1)$ : the conclusion of the theorem follows from Wall [W], Theorem E, p. 63. It follows easily from Poincaré duality that $S_{*}(\widetilde{X})$ and $S_{*}(\widetilde{X}, \partial \widetilde{X})$ satisfy $H D(n)$. Moreover $G=\pi_{1}(X)$ is finitely generated [Brd] (Corollary 1', p. 195), so (4.1) applies. 
First consider the case $\partial X=\varnothing$ and let $\mathcal{D}=H^{n}\left(S_{*}(\tilde{X}) ; \mathbb{Z} G\right)$. Since $X$ is a connected Poincaré duality space, there is a fundamental class $[X] \in H_{n}\left(S_{*}(\widetilde{X}) ; \mathcal{D}\right)$ such that the cap product with $[X]$ yields the usual isomorphisms. In particular, for any left $\mathbb{Z} G$ module $M$, the cap product by $[X]$ yields an isomorphism

$$
\operatorname{ev}_{M}: H^{n}\left(S_{*}(\tilde{X}) ; M\right) \rightarrow H_{0}\left(S_{*}(\tilde{X}) ; \mathcal{D} \otimes_{\mathbb{Z}} M\right)=\mathcal{D} \otimes_{\mathbb{Z} G} M
$$

The diagram

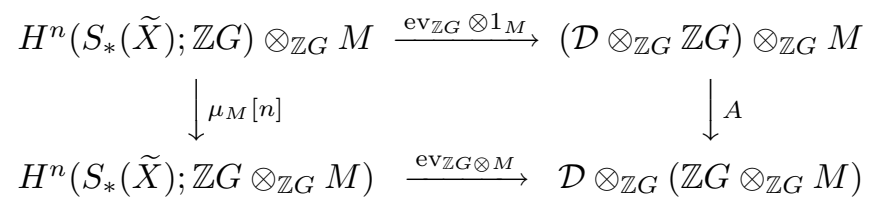

commutes, where $A$ is the usual associativity isomorphism. It follows that the $\mu_{M}[n]$ are isomorphisms which is one of the hypotheses of Proposition 1 . Since $X$ is connected, $\mathcal{D}=\mathbb{Z}$ as $\mathbb{Z}$ modules, and (5.1) verifies the hypotheses of (5.2). Hence (5.2) verifies the remaining hypothesis of Proposition 1 and Theorem A follows.

If $\partial X \neq \varnothing$, then Theorem $\mathrm{A}$ has two possible interpretations. It follows easily from Poincaré duality that $S_{*}\left(\left.\widetilde{X}\right|_{H}\right)$ satisfies $H D(n-1)$ for any subgroup $H$, so the more interesting result is that $S_{*}\left(\left.\widetilde{X}\right|_{H},\left.\partial \widetilde{X}\right|_{H}\right)$ satisfies $H D(n-1)$ if $H$ has infinite index in $G$. The proof is a straightforward generalization of the closed case.

Proof of Proposition 1. The Shapiro Lemma [Brn] (p. 73) says $H^{r}\left(R H \mid E_{*} ; L\right) \cong$ $H^{r}\left(E_{*} ; \operatorname{Hom}_{R H}(R G, L)\right)$. The first statement follows if these cohomology groups are 0 for $r \geq n$. For $r>n$ this follows from $H D(n)$. For $r=n, H^{n}\left(E_{*} ; R G\right) \in$ $\mathcal{C}_{H}{ }^{R}{ }_{G}$ implies $H^{n}\left(E_{*} ; R G\right) \otimes_{R G} \operatorname{Hom}_{R H}(R G, L)=0$. But $\mu[n]: H^{n}\left(E_{*} ; R G\right) \otimes_{R G}$ $\operatorname{Hom}_{R H}(R G, L) \rightarrow H^{n}\left(E_{*} ; \operatorname{Hom}_{R H}(R G, L)\right)$ is onto, so $R H \mid E_{*}$ satisfies $H D(n-1)$. Turning to the second half of Proposition 1, if $R H \mid E_{*}$ satisfies $H D(n-1)$, then $H^{n}\left(E_{*} ; \operatorname{Hom}_{R H}(R G, L)\right)=0$, so the injectivity of $\mu_{K}[n] \operatorname{implies} H^{n}\left(E_{*} ; R G\right) \otimes_{R G}$ $\operatorname{Hom}_{R H}(R G, L)=0$.

Proof of Proposition 2. Clearly (2.3) implies (2.2) implies (2.1). Composition, $c$ : $\operatorname{Hom}_{R H}(R G, R G) \otimes_{R G} \operatorname{Hom}_{R H}(R G, L) \rightarrow \operatorname{Hom}_{R H}(R G, L)$, is a left $R G$ module map. The identity from $D \otimes_{R G} \operatorname{Hom}_{R H}(R G, L)$ to itself factors as

$\lambda_{H} \otimes 1: D \otimes_{R G} \operatorname{Hom}_{R H}(R G, L) \rightarrow \mathcal{D} \otimes_{R G} \operatorname{Hom}_{R H}(R G, R G) \otimes_{R G} \operatorname{Hom}_{R H}(R G, L)$ composed with

$1 \otimes c: \mathcal{D} \otimes_{R G} \operatorname{Hom}_{R H}(R G, R G) \otimes_{R G} \operatorname{Hom}_{R H}(R G, L) \rightarrow \mathcal{D} \otimes_{R G} \operatorname{Hom}_{R H}(R G, L)$.

This shows (2.1) implies (2.3).

Proof of Proposition 3. Results (3.3) and (3.4) follow since the tensor product is right exact. Result (3.5) follows since tensor product preserves sums.

If $H \subset G^{\prime} \subset G$, define $\Lambda: R G \otimes_{R G^{\prime}} \operatorname{Hom}_{R H}\left(R G^{\prime}, L\right) \rightarrow \operatorname{Hom}_{R H}(R G, L)$ by $\Lambda(x \otimes f)(y)=f\left((y x)_{G^{\prime}}\right)$ where $(y x)_{G^{\prime}}=\sum_{k \in G^{\prime}} r_{k} k$ if $y x=\sum_{g \in G} r_{g} g$. Note $\Lambda$ is a map of left $R G$ modules. If $\left|G: G^{\prime}\right|<\infty$, we construct an inverse for $\Lambda$ as follows. Let $\mathcal{S}=\left\{x_{i} \in G\right\}$ be a set of coset representatives for $G^{\prime} \backslash G$ and define $\Psi_{\mathcal{S}}: \operatorname{Hom}_{R H}(R G, L) \rightarrow R G \otimes_{R G^{\prime}} \operatorname{Hom}_{R H}\left(R G^{\prime}, L\right)$ by $\Psi_{\mathcal{S}}(f)=\left.\sum_{i} x_{i}^{-1} \otimes\left(x_{i} \cdot f\right)\right|_{G^{\prime}}$, where here $\left.\left(x_{i} \cdot f\right)\right|_{G^{\prime}}$ denotes the homomorphism restricted to $R G^{\prime}$. Check that $\Psi_{\mathcal{S}}$ is the inverse to $\Lambda$, so $D$ is an $R G$ module in $\mathcal{C}_{H}{ }^{R}{ }_{G}$ iff $\left.D\right|_{R G^{\prime}} \in \mathcal{C}_{H}{ }^{R} G^{\prime}$. Result (3.1) follows. 
Let $H^{\prime} \subset H \subset G$. Since $\operatorname{Hom}_{R H}(R G, R G) \subset \operatorname{Hom}_{R H^{\prime}}(R G, R G),(2.1)$ proves $\mathcal{C}_{H}{ }^{R}{ }_{G} \subset \mathcal{C}_{H^{\prime}}{ }^{R}{ }_{G}$. If $\left|H: H^{\prime}\right|<\infty$, choose coset representatives $x_{i}$ for $H^{\prime} \backslash H$, and define $\tau: \operatorname{Hom}_{R H^{\prime}}(R G, R G) \rightarrow \operatorname{Hom}_{R H}(R G, R G)$ by $\tau(f)(y)=\sum_{x_{i}} x_{i}^{-1} f\left(x_{i} y\right)$. Check $\tau$ is a left $R G$ module map. Choose coset representatives, $g_{\alpha}$, for $H \backslash G$. Define

$$
v(g)= \begin{cases}g & \text { if } g \in H^{\prime} g_{\alpha} \text { for some } \alpha, \\ 0 & \text { if } g \notin H^{\prime} g_{\alpha} \text { for any } \alpha .\end{cases}
$$

Check $v \in \operatorname{Hom}_{R H^{\prime}}(R G, R G)$ and $\tau(v)=1_{R G}$. It follows that $\lambda_{H}$ factors through $D \otimes_{R G} \operatorname{Hom}_{R H^{\prime}}(R G, R G)$ so (2.2) and (2.1) prove $\mathcal{C}_{H^{\prime}}{ }^{R}{ }_{G} \subset \mathcal{C}_{H}{ }^{R}{ }_{G}$. Result (3.2) follows.

Proof of Proposition 5. Result (5.1) holds since the $R$ module automorphisms of $R$ are $R^{\times}$. To prove the remaining results, let $\omega: G \rightarrow R^{\times}$be a homomorphism, and let $\omega: R G \rightarrow R G$ be the ring homomorphism defined by $\omega\left(\sum_{g \in G} r_{g} g\right)=$ $\sum_{g \in G} r_{g} \omega(g) g$. Given any right $R G$ module $D$, let $D^{\omega}$ denote $D$ with a new $R G$ module structure given by $d \cdot \omega x=d \cdot \omega(x)$. Given $f \in \operatorname{Hom}_{R H}(R G, R G)$ define $f^{\omega}(x)=\omega\left(f\left(\omega^{-1}(x)\right)\right)$ and check that $f^{\omega} \in \operatorname{Hom}_{R H}(R G, R G)$. Define $\Lambda$ : $D^{\omega} \otimes_{R G} \operatorname{Hom}_{R H}(R G, R G) \rightarrow D \otimes_{R G} \operatorname{Hom}_{R H}(R G, R G)$ by $\Lambda(d \otimes f)=d \otimes f^{\omega}$. Note $\Lambda$ is an isomorphism. Since $1_{R G}^{\omega}=1_{R G}$, it follows from (2.1) that

$$
D \in \mathcal{C}_{H}^{R}{ }_{G} \quad \text { iff } \quad D^{\omega} \in \mathcal{C}_{H}{ }_{G} .
$$

Finally, we show (5.2) and (5.3). Let $H_{D}=H \cap G_{D}$ and note $H_{D}$ has finite index in $H$. It follows from (3.1) and (3.2) that we may assume $G=G_{D}$ without loss of generality. By (3.6) in case (5.3) we may assume further that $G$ is free. Under either of our two assumptions, $s: G \rightarrow \operatorname{Aut}^{\mathrm{op}}(D)$ factors through a homomorphism $\omega: G \rightarrow R^{\times}$. If $D^{\operatorname{tr}}$ denotes $D$ as an $R$ module but with trivial $G$ action, $D=$ $\left(D^{\operatorname{tr}}\right)^{\omega}$. From (5.4), we need only prove $D^{\operatorname{tr}} \in \mathcal{C}_{H}{ }^{R}{ }_{G}$. This follows from (3.5), from (3.3) and from the assumption that $R^{\text {tr }} \in \mathcal{C}_{H}{ }^{R} G$.

Remarks 1. 1. Strebel [St], p. 324, shows that $\mathbb{Z}^{\text {tr }} \notin \mathcal{C}_{e}{ }^{\mathbb{Z}}{ }_{G}$ whenever $G$ is infinite but locally finite. His technique generalizes to show a partial converse for (4.2): if for every finitely generated subgroup $G^{\prime} \subset G$ there exists a $g \in G$ such that the index of $G^{\prime} \cap g \mathrm{Hg}^{-1}$ in $G^{\prime}$ is finite, then $\mathbb{Z}^{\operatorname{tr}} \notin \mathcal{C}_{H} \mathbb{Z}_{G}$.

2. For duality groups in the sense of Bieri and Eckmann [Brn], pp. 219-225, $\mu_{M}[n]$ is still an isomorphism, so Proposition 1 implies that, for any subgroup $H$ of a duality group $G$,

$$
K(H, 1) \text { satisfies } H D(n-1) \quad \text { iff } \quad H^{n}(G ; \mathbb{Z} G) \in \mathcal{C}_{H}{ }_{G} .
$$

As Strebel remarks, there are examples of duality groups and subgroups for which $|G: H|=\infty$ but $K(H, 1)$ does not satisfy $H D(n-1)$. This shows that more than infinite index is required in general for the dimension to drop. The only if part of (6) also gives restrictions on the dualizing module: e.g., since $K(\{e\}, 1)$ satisfies $H D(0), H^{n}(G ; \mathbb{Z} G) \otimes_{\mathbb{Z} G} \operatorname{Hom}_{\mathbb{Z}}(\mathbb{Z} G, \mathbb{Z} G)=0$.

\section{REFERENCES}

[Brd] W. Browder, Poincaré spaces, their normal fibrations and surgery, Invent. Math. 17 (1972), 191-202. MR 48:5086

[Brn] K. S. Brown, Cohomology of groups, Graduate Texts in Math., vol. 87, Springer-Verlag, New York, 1982. MR 83k:20002 
[CE] H. Cartan and S. Eilenberg, Homological algebra, Princeton Univ. Press, Princeton, NJ, 1956. MR 17:1040e

[H] J. A. Hillman, A homotopy fibration theorem, Topology Appl. 33 (1989), 151-161. MR 90k:57023

[St] R. Strebel, A remark on subgroups of infinite index in Poincaré duality groups, Comment Math. Helv. 52 (1977), 317-324. MR 56:15793

[W] C. T. C. Wall, Finiteness conditions for CW complexes, Ann. of Math. (2) 81 (1965), 56-69. MR 30:1515

Department of Mathematics, University of Notre Dame, Notre Dame, Indiana 46556

E-mail address: dwyer.1@nd.edu

E-mail address: stolz.1@nd.edu

E-mail address: taylor.2@nd.edu 